\title{
UTILITY OF CONE BEAM COMPUTED TOMOGRAPHY OF THE MANDIBLE IN DETECTION OF OSTEOPOROSIS IN POSTMENOPAUSAL WOMEN
}

\author{
Noha M. Elkersh ${ }^{1} B D S, P h D$, Maha R.Talaab² PhD, Walid M. Ahmed ${ }^{3}$ PhD, Yousria S. \\ Gaweesh ${ }^{4} P h D$
}

\begin{abstract}
INTRODUCTION: Osteoporosis is a systemic disease characterized by decreased bone mass and increased bone fragility. Dental radiographs are widely used in the adult population especially Cone Beam Computed Tomography (CBCT) that is used in oral and maxillofacial surgery for numerous clinical applications. Therefore, dental radiographs may offer an opportunity as a screening tool for the early detection of osteoporosis and subsequent referral.

OBJECTIVES: The aim of this study was to evaluate the diagnostic efficacy of mandibular CBCT images for the detection of osteoporosis in postmenopausal women and to correlate it to Dual energy x-ray absorptiometry (DEXA) scan results.

MATERIALS AND METHODS: twenty four postmenopausal female patients were recruited in this study. Based on their DEXA results, they were classified into osteoporotic and control non-osteoporotic groups. CBCT examination was done as part of surgical treatment. Coronal images were used to measure the computed tomography mandibular index (CTMI) and the computed tomography index (superior) (CTIS).The correlation between these indices and bone mineral density (BMD) measured by DEXA were assessed.

RESULTS: There were significant differences between the control and osteoporotic groups in the computed tomography mandibular index superior CTI(S), and computed tomography mental index (CTMI: inferior cortical width).There was significant positive correlation between CTMI and T-score measured by DEXA.
\end{abstract}

CONCLUSIONS: CTI(S) and CTMI on the CBCT images can be used to assess the osteoporotic women.

KEYWORDS: Cone-Beam Computed Tomography; Bone Density; Osteoporosis

1-Instructor at Oral Medicine, Periodontology, Oral Diagnosis and Radiology Department, Faculty of Dentistry, Alexandria University, Alexandria, Egypt.

2-Lecturer of Oral Medicine, Periodontology, Oral Diagnosis and Radiology Department, Faculty of Dentistry, Alexandria University, Alexandria, Egypt.

3-Lecturer of Radio diagnosis, Medical Research Institute, Alexandria University

4-Professor of Oral Medicine, Periodontology, Oral Diagnosis and Radiology Department, Faculty of Dentistry, Alexandria University, Alexandria, Egypt

Corresponding author:

E-mail: nohamohamedelkersh@gmail.com

\section{INTRODUCTION}

Osteoporosis is a serious skeletal disorder that weakens bones and increases the risk of developing fractures (1). It is characterized by a low bone mass, deterioration of the bone structure and increased bone fragility (2). Moreover, it is considered a priority health problem because it affects more than 20 million people, most of them women, worldwide. It causes over 2 million bone fracture incidents annually. Women after menopause are more commonly affected; an estimate of $33.33 \%$ of women and $12.5 \%$ of men over 50 years old reported (3). This disorder affects women more than cancer, heart disease and stroke combined (1). The problem with osteoporosis is that no signs of the disease are manifested until a fragility fracture occurs. For this reason, intense interest exists within the medical community for developing accurate early diagnostic techniques $(4,5)$.

The World Health Organization (WHO) in 1994 introduced a new epidemiological definition of osteoporosis and osteopenia, based on measurements of bone mineral density (BMD) expressed in Standard Deviation units called T-scores. Osteoporosis and osteopenia were defined by Tscore thresholds of equal to or less than 2.5 or $1.0 \mathrm{SD}$ respectively, below the healthy young adult mean (6).

Osteoporosis can be classified as a primary or secondary disease. Primary osteoporosis is the most common form and includes two categories: in postmenopausal women "postmenopausal" osteoporosis or in older men and women "senile" osteoporosis as human bones decrease in density and increase in porosity beginning at about the third decade of life (7). There is an intimate relationship between reproductive hormone deficiency and the development of postmenopausal osteoporosis (8).

The term "secondary" osteoporosis refers to bone loss resulting from specific, defined clinical disorders, such as hyperparathyroidism or hyperadrenocorticism (8).

The effect of general mineral loss of skeleton, osteoporosis, on the jawbones has been extensively studied, especially with new radiologic methods (7). Osteoporosis may affect the structure of the alveolar bone (3). Several investigators have stated that osteoporosis results in reduced jaw bone mass and altered mandibular structure, especially of the inferior border (mandibular lower cortex) (9), this may interfere with implant placement and other dental procedures (3).

Jaw bone density can be assessed using intraoral and panoramic radiographs, cone beam computed tomography (CBCT), quantitative computed tomography (QCT), and dual energy X-ray absorptiometry (DEXA).DEXA is known as the most accurate clinical method for identifying those with low bone mineral density (BMD) (2). However; BMD testing, especially DEXA, for all postmenopausal women is not feasible in many countries. Moreover, most of the post- menopausal women have more opportunities to 
visit a dental clinic for the treatment of dental caries and periodontal disease, than to visit a medical office for diagnosis of osteoporosis prior to fracture (10).

Measurement of the cortical width and a subjective assessment of cortical porosity on panoramic radiographs are methods that were previously used to diagnose osteoporosis (11-13).

Nowadays CBCT is used in oral maxillofacial radiology for numerous clinical applications, particularly for its low radiation compared with multi-slice computerized tomography (14).

Such radiographs have the added advantage of a high requirement need for treatment planning compared to the rest of the body, and less full body radiation exposure, so it would be useful to know whether radiographic changes in mandible could have a role in detection of osteoporosis. This would put the dentist in a position to identify such patients, modify the treatment plan and refer them for prompt management (15).

Therefore, in order to evaluate the diagnostic efficacy of CBCT images in the detection of osteoporosis, CBCT technique was used to assess CT indices for postmenopausal osteoporotic females and correlate them with their body bone mineral density as detected by DEXA scan.

\section{MATERIALS AND METHODS}

An approval was obtained from the ethics committee at the faculty of dentistry, Alexandria University and all patients were asked to sign an informed consent before the commence of the study.

\section{Study design}

The study was conducted on twenty four female patients with ages above 45 years,selected from the outpatient clinic of the Department of Oral Medicine, Periodontology, Oral Diagnosis, and Oral Radiology, Faculty of Dentistry, Alexandria University.

\section{Sample Size}

A sample of 24 post menopausal females(16) was required to estimate an average difference at computed tomography mandibular index superior (CTIS) measures between normal and osteoporotic females $=0.07 \mathrm{~mm}(2), \mathrm{SD}=$ $0.05 \mathrm{~mm}$ for both (based on DEXA scan as standard), using alpha error $=0.05$ provided a study power of $80 \%$.

The sample was selected randomly using simple random sample technique.

The sample size was calculated using Med Calc software.

\section{Criteria for patient selection \\ Inclusion Criteria}

1- Patients age over 45 years.

2- Postmenopausal women.

3- Women with a history of amenorrhea for at least five years.

4- Ambulatory patients

5- Patients indicated for CBCT for dental surgical procedures.

\section{Exclusion Criteria}

1- Patients suffering from metabolic bone diseases (hyper, hypoparathyroidism, Paget disease, osteomalacia).

2- Patients with any systemic disease that affects bone metabolism
3- History of smoking or tobacco use.

4- History of using any drug that affects bone metabolism within the past 6 months.

\section{Radiographic examination:}

\section{Evaluation of bone mineral density}

The BMDs (T-score) of the lumbar vertebrae (L1-L4) and femur were calculated using a DEXA scanner (LUNAR Prodigy machine). The height and weight were measured at the time of DEXA scanning.

The twenty four patients were classified into two groups, according to the T-score defined by the World Health Organization (WHO) (17):

Group 1: Control group consisted of thirteen patients classified as non osteoporotic with respect to bone mineral density (BMD) (T-score $>-2.5)$;

Group 2: Test group consisted of eleven patients classified as osteoporotic with respect to bone mineral density (BMD) (T-score<-2.5).

\section{CBCT images}

CBCT images of the mandible were obtained using I-CAT machine (Imaging science International, 2nd generation, Hatfield, PA, USA). Scans were set at $120 \mathrm{kV}, 37 \mathrm{~mA}, 26.9$ seconds, voxel size of $0.25 \mathrm{~mm}$ and field of view $16 \mathrm{x} 6 \mathrm{~cm}$. Slice selection

Using the multiplanar reformation screen (MPR) using On Demand software (Cyber med Inc, Korea), axial images with a slice thickness of $0.1 \mathrm{~mm}$ were selected and the mental foramen was identified by scrolling through sequential slices.

The slice which clearly identified and represented the widest mesiodistal dimension of the mental foramen was selected. Two indices were assessed on the CBCT images. As CBCT was utilized in this study, the terms of the indices used were obtained as a result of a modification of Ledgerton's classification for panoramic images $(11,18)$.

The indices were: "CTI(S)" and "CTMI"

CTI(S): computed tomography mandibular index (superior), which is the ratio of the inferior cortical width to the distance from the superior margin of the mental foramen to the inferior border of the mandible. CTI (S) is related to the PMI. The PMI is a radiometric measurement introduced by Benson et al. (19) and is the ratio between the mandibular cortical bone thickness and the distance from the superior margin of the mental foramen to the inferior border of the mandible.

$\mathrm{CTI}(\mathrm{S})=\mathrm{W} / \mathrm{S}$

CTMI: computed tomography mental index, which is the inferior cortical width of the mandible and symbolized the mental index (MI) as described by Ledgerton et al. (18) $\mathrm{CTMI}=\mathrm{W}$

On the axial view at the mental foramen region, the $\mathrm{Y}$-axis (arrow) has been changed to be tangent to the outer border of mandible (figure 1). 


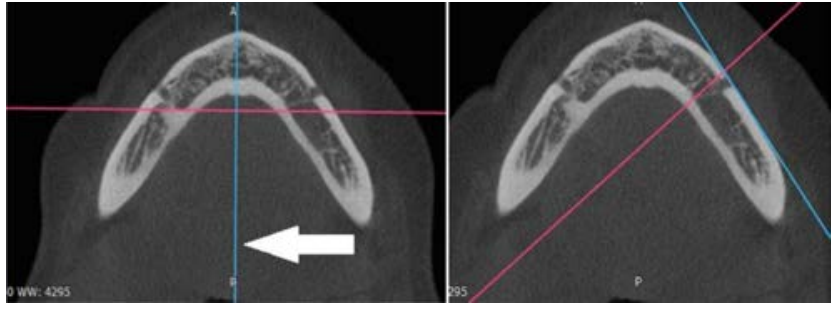

Figure(1): Slice adjustment in axial view

On the coronal view the same $\mathrm{Y}$-axis has been moved to the bisecting position of the bucco-lingual dimension of the mandibular body and it has been changed simultaneously in the axial view (figure 2).
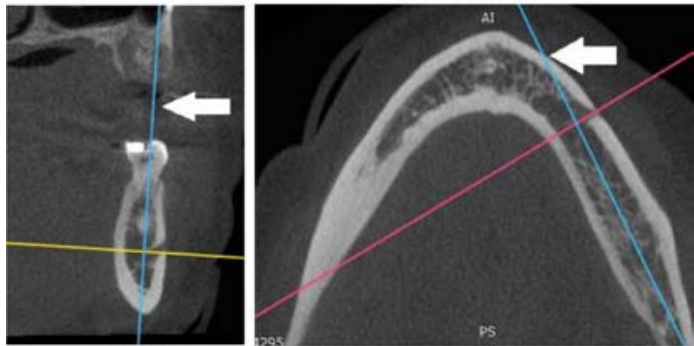

Figure(2): Slice adjustment in coronal view

On the sagittal view, the Z-axis (arrow) has been moved to be parallel with the inferior border of the mandible (figure 3).

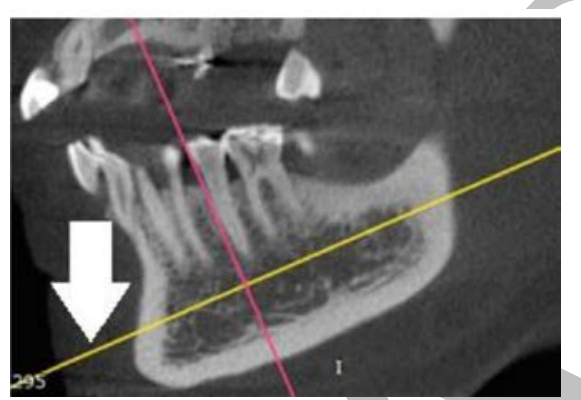

Figure(3): Slice adjustment in sagittal view

Measurements were taken from the coronal view (figure 4).
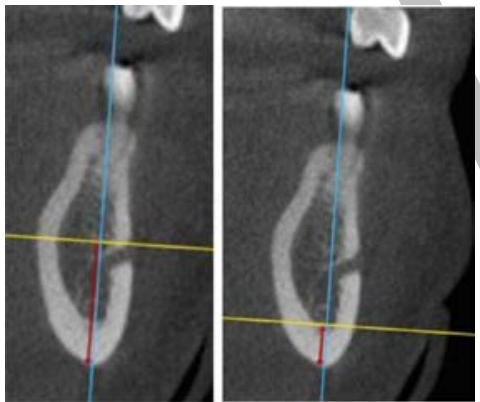

Figure(4): CBCT measurements

\section{Statistical analysis}

Data was fed to the computer and analyzed using IBM SPSS software package version 20.0. (IBM Corp, Armonk, NY, USA). Qualitative data were described using number and percent. The Kolmogorov-Smirnov test was used to verify the normality of distribution. Quantitative data were described using range (minimum and maximum), mean, standard deviation and median. Significance of the obtained results was judged at the 5\% level. Differences were analyzed using t-test.

\section{RESULTS}

The present study was carried out to evaluate the utility of CBCT in the detection of osteoporosis in postmenopausal women.

\section{Clinical data of the subjects:}

In this study, 24 female patients were recruited and were divided into 2 groups according to the T-score defined by the World Health Organization (WHO):

Group 1: consisted of control non osteoporotic patients and included two subgroups:

o normal patients with T-score $>-1$

o osteopenic patients with $(-1>\mathrm{T}$-score $>-2.5)$.

$(-1>$ T-score $>-2.5)$.

Group 2: Osteoporotic women (T-score $<-2.5)$.

Table-1 shows the demographic data of the recruited patients of the studied groups regarding age.

Table (1): Descriptive analysis of the studied groups according to age

\begin{tabular}{|c|c|c|c|c|c|}
\hline & \multicolumn{2}{|c|}{ Control } & \multirow{2}{*}{$\begin{array}{c}\text { Osteoporo } \\
\text { tic }(n=11)\end{array}$} & \multirow[b]{2}{*}{$\mathbf{F}$} & \multirow[b]{2}{*}{$\mathbf{P}$} \\
\hline & $\begin{array}{c}\text { Normal } \\
(n=6)\end{array}$ & $\begin{array}{c}\text { Osteopeni } \\
\text { a }(n=7)\end{array}$ & & & \\
\hline Age (years) & & & & & \\
\hline Min. - Max. & $45.0-56.0$ & $47.0-65.0$ & $52.0-68.0$ & & \\
\hline Mean \pm SD & $49.50 \pm 4.37$ & $56.0 \pm 7.21$ & $\begin{array}{c}57.73 \pm \\
5.66\end{array}$ & 3.390 & 0.079 \\
\hline Median & 49.0 & 59.0 & 56.0 & & \\
\hline
\end{tabular}

F,p: $\mathrm{F}$ and $\mathrm{p}$ values for ANOVA test

\section{Radiographic examination}

\section{DEXA scanning}

Figures 5 and 6 show the descriptive analysis of the studied groups according results of DEXA scan.

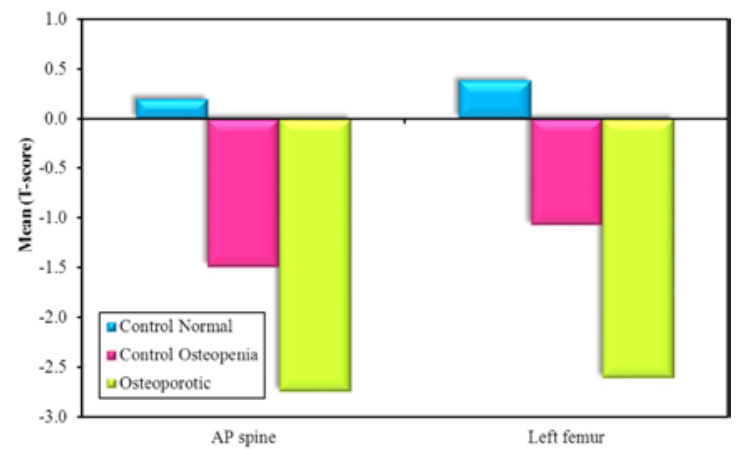

Figure (5): Descriptive analysis of the studied groups according to AP spine (T-score) and Left femur (T-score) of DEXA scanning

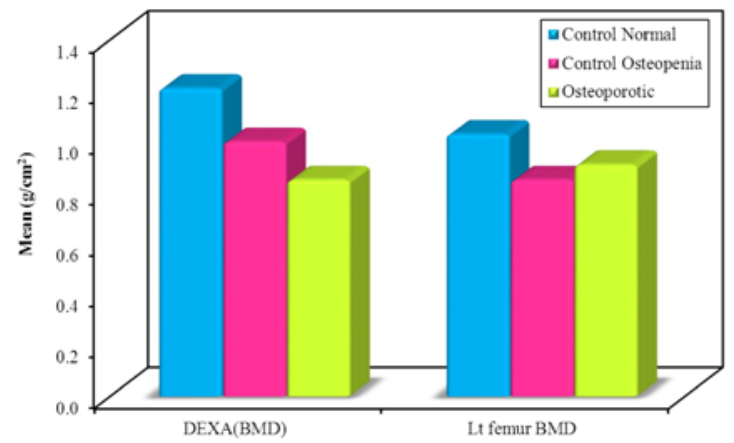

Figure (6): Descriptive analysis of the studied groups according to DEXA (BMD) $\left(\mathrm{g} / \mathrm{cm}^{2}\right)$ and Lt femur BMD $\left(\mathrm{g} / \mathrm{cm}^{2}\right)$ of DEXA scanning 


\section{CBCT indices:}

As a result of the small sample size of the study comparison was done between the two main groups only osteoporotic group and non-osteoporotic (control) group.

Table 2 illustrates comparison between the two studied groups according to CBCT indices.

Table (2): Comparison between the two studied groups according to CBCT parameters

\begin{tabular}{|c|c|c|c|c|}
\hline CBCT & Control $(n=13)$ & $\begin{array}{c}\text { Osteoporosis } \\
(n=11)\end{array}$ & t & $\mathbf{P}$ \\
\hline $\begin{array}{c}\text { CTI (S) } \\
\text { Min. - Max. } \\
\text { Mean } \pm \text { SD. } \\
\text { Median }\end{array}$ & $\begin{array}{c}0.22-0.35 \\
0.27 \pm 0.04 \\
0.27\end{array}$ & $\begin{array}{c}0.16-0.29 \\
0.20 \pm 0.04 \\
0.19\end{array}$ & $\begin{array}{c}3.74 \\
8^{*}\end{array}$ & $\begin{array}{c}0.00 \\
1^{*}\end{array}$ \\
\hline $\begin{array}{c}\text { CTMI } \\
\text { Min. - Max. } \\
\text { Mean } \pm \text { SD. } \\
\text { Median }\end{array}$ & $\begin{array}{c}3.50-5.20 \\
4.12 \pm 0.53 \\
4.0\end{array}$ & $\begin{array}{c}2.69-4.50 \\
3.44 \pm 0.63 \\
3.44\end{array}$ & $\begin{array}{c}2.85 \\
4^{*}\end{array}$ & $\begin{array}{c}0.00 \\
9^{*}\end{array}$ \\
\hline
\end{tabular}

$\mathrm{t}, \mathrm{p}$ : $\mathrm{t}$ and $\mathrm{p}$ values for Student t-test for comparing between the two groups

*: Statistically significant at $\mathrm{p} \leq 0.05$

The mean values for CTI(S) were 0.27 in control group and 0.19 in osteoporosis group while the mean values for CTMI were 4.12 in control group, and 3.15 in osteoporosis group. On comparing the studied groups, a statistically significant difference was detected between the control and osteoporosis groups regarding CTI(S) and CTMI $(\mathrm{p}<0.05)$. The control group showed higher mean values than the osteoporotic group.

\section{Correlation between CBCT indices and DEXA:}

Table 3 shows the correlation between CBCT indices and T-score as measured by DEXA scan.

Table (3): Correlation between CBCT with different parameters in each group

\begin{tabular}{|c|c|c|c|c|}
\hline \multicolumn{1}{|c|}{} & \multicolumn{3}{|c|}{} \\
\cline { 2 - 5 } & \multicolumn{2}{|c|}{ CTI (S) } & \multicolumn{2}{c|}{ CTMI } \\
\cline { 2 - 5 } & $\mathbf{r}$ & $\mathbf{p}$ & $\mathbf{r}$ & $\mathbf{P}$ \\
\hline Control (n= 13) & & & & \\
\hline AP spine & 0.462 & 0.112 & $0.706^{*}$ & $0.007^{*}$ \\
\hline Left femur & 0.448 & 0.125 & $0.676^{*}$ & $0.011^{*}$ \\
\hline Osteoporosis (n=11) & & & & \\
\hline AP spine & $0.673^{*}$ & $0.023^{*}$ & $0.851^{*}$ & $0.001^{*}$ \\
\hline Left femur & $-0.707^{*}$ & $0.015^{*}$ & -0.298 & 0.373 \\
\hline Total cases (n= 24) & & & & \\
\hline AP spine & $0.853^{*}$ & $<0.001^{*}$ & $0.856^{*}$ & $<0.001^{*}$ \\
\hline Left femur & $0.814^{*}$ & $<0.001^{*}$ & $0.811^{*}$ & $<0.001^{*}$ \\
\hline
\end{tabular}

r: Pearson coefficient

$*$ : Statistically significant at $\mathrm{p} \leq 0.05$

CTMI showed strongest positive correlation with Tscore of lumbar spine in both control and osteoporotic group ( $\mathrm{r}=0.7$ and 0.8 respectively) followed by CTIS which showed moderate positive correlation in control group $(\mathrm{r}=0.4)$ and strong positive correlation in osteoporotic group $(\mathrm{r}=0.6)$.

On the other hand, regarding correlation with T-score of left femur only CTMI in control group showed strong positive correlation $(\mathrm{r}=0.6)$ while other index showed no correlation.

\section{DISCUSSION}

Osteopenia and osteoporosis affect many aged individuals and might not be detected until symptoms or fractures occur. Early detection of the disease is important to maximize bone mass retention, reduce the risk of fracture, and avoid subsequent pain in these patients $(20,21)$.

The silent nature of this disease increases the difficulty of its diagnosis. Therefore, all medical professionals should participate in identifying patients suffering from osteoporosis and refer suspicious cases to specialists (22).

As osteoporotic bone loss affects various parts of the body, it includes also the jaws (21). Researchers have demonstrated a positive correlation between BMD in the mandible and that in hip and spine (23). It is also evident that the reduction of skeletal BMD can alter mandibular shape; raising the possibility that osteoporosis may be associated with specific shape changes in this bone (21).

Postmenopausal women with severely eroded mandibular cortices or with substantial thinning of the cortices present lower BMD values (24).

Panoramic mandibular radiomorphometric indices were proved to be one of the preliminary osteoporosis diagnostic tools (25).

However, measurement of the thickness of lower mandibular cortical bone in panoramic radiography is limited by the difficulty of precisely locating the mental foramen and determining the upper border of the mandibular cortical bone $(26,27)$.

These limitations may be overcome by CBCT, because tomographic images make it possible to precisely locate and view the mental foramen.

In the present study CBCT was used as an evaluation tool as it provides three-dimensional images of high resolution. It also allows the qualitative and quantitative evaluation of osseous structures (4). The results obtained by CBCT were compared to those of the DEXA scan, which is considered to be the gold standard for diagnosing osteoporosis (28-30). Based on DEXA results, two groups comprising the osteoporosis group and the non osteoporosis control group were defined.

Our results showed a highly significant difference between the two study groups for CTIS and CTMI scores $(p<0.05)$. Moreover, the mean values of these indices showed higher values in the non-osteoporotic group than in the osteoporotic group.

Our results are in agreement with the few studies that could be found in the dental literature that investigated these indices using CBCT. A study by Koh and Kim (11) who were the first to evaluate CBCT as a low-BMD predictor, showed that there was a significant difference between the normal and osteoporotic groups regarding CTIS and CTMI. Moreover, these indices showed higher mean values in healthy rather than in osteoporotic group.

Another study was done by Mostafa et al. (4) where they showed similar results in their study, significant differences for the CT mental index (CTMI) and CT mandibular index (CTI) between the control and osteoporotic groups were found, whereby the control group showed higher mean values than the osteoporotic one.

Furthermore, a similar study was done by Güngör et al. (28). They found that the paired comparison of osteoporosis and osteopenia groups and of the osteoporosis and control groups showed significant differences in all index 
measurements (CTIS and CTMI), while the paired comparison of the osteopenia and control group revealed no significant differences in index measurements. Their findings indicated that in the presence of osteopenia, no changes in the jaw indices were apparent, whereas in the presence of osteoporosis, significant differences were detected in the index measurements.

In the present study we made a comparison between the control group (including both normal and osteopenic patients) and osteoporosis group only as we found no changes in jaw bones in osteopenia group.

Similarly in a study by Brasileiro et al. (27) DEXA was performed, and the patients were divided into normal, osteopenia, and osteoporosis groups, according to the World Health Organization (WHO) criteria. Cross-sectional images were used to evaluate the computed tomography indices. Mean CTMI and CTI (S) values were lower in the osteoporosis group than in osteopenia and normal patients $(\mathrm{p}<0.05)$.

In our study regarding correlation between CBCT indices and T-score measured by DEXA a high significant positive correlation was found between CTMI and lumbar spine T-score $(\mathrm{p}=0.007),(\mathrm{r}=0.7)$ in control group and $(\mathrm{r}=0.8)$ in osteoporosis group ; while CTI(S) showed moderate positive correlation $(\mathrm{r}=0.4)$ in control group and $(\mathrm{r}=0.6)$ in osteoporosis group only.

This can be explained by the fact that CTMI is a direct measurement index which is the thickness of mandibular cortex, while CTI(S) index is ratio and subsequently depends on the anatomical location of mental foramen.

In our study, DEXA was performed at two different areas, namely lumbar vertebrae (spine) and proximal femur as the rate of bone turnover and bone loss is not uniform throughout the body (31). Our results showed no correlation with T-score of left femur.

This can be explained by the fact that sites rich in trabecular bone, such as the vertebrae, are likely to be affected earlier after the menopause and to a greater degree than sites rich in cortical bone such as the femoral neck .This may explain some of the discordance in T-scores when different sites are compared (32). Therefore, in osteoporosis evaluations, the measurement of vertebral BMD is preferred in postmenopausal females (as in our study) while measurement of the femoral head BMD is favored in individuals over 70 years (28).

Corresponding results were reported by Güngör et al. (28) showing positive correlation between vertebral BMD and CTMI and CTI(S).

However, the study done by Mostafa et al. (4) showed a highly significant positive correlation between all indices and lumbar spine BMD measured by DEXA (T-score). This significant correlation could be as result of larger sample size used in their previous study.

As a result of limited studies using CBCT for the assessment of radiomorphomertic indices and their correlations with BMD measured by DEXA, our study results were compared with the results of similar studies that used panoramic images.

Studies that evaluated the correlation between panoramic mandibular index (PMI) in panoramic radiography that corresponds to $\mathrm{CTI}(\mathrm{S})$ in CBCT and BMD showed controversial results. Some authors found a significant correlation between PMI and BMD as Singh et al. (33) and Passos et al. (34). The former found that mean BMD scores increased significantly with increasing PMI ( p <0.05) so , significant correlations between PMI and DEXA BMD were obtained. Also Passos et al. (34) observed that the mandibular cortical width (MCW corresponding to CTMI in CBCT) presented a significant positive correlation at both of the sites (left femur and lumbar spine) and that the PMI correlated with lumbar spine only.

On the other hand, others did not find this correlation such as Damilakis and Vlasiadis (35) who found weak correlations between mandibular cortical width (MCW) and BMD values and no correlation between PMI and BMD. Moreover, they concluded that MCW was better than PMI ratio in its power to differentiate women with osteopenia or osteoporosis from healthy subjects.

These conflicting results could be as a result of different populations under studies on one hand and different machines used in the studies on the other.

\section{CONCLUSION}

The results of this study suggest that quantitative and qualitative CBCT indices may be useful in identifying postmenopausal women with low BMD and as a screening tool for osteoporosis. These indices are able to help dentists screen women with low spinal and femoral bone mineral density so that they can refer them for bone densitometry. Further research should contain a larger sample size of female populations of different age groups and menstruation conditions.

\section{CONFLICT OF INTEREST}

The authors declare that they have no conflicts of interest.

\section{REFERENCES}

1. For the dental patient. Osteoporosis medications and the risk of developing jaw complications. J Am Dent Assoc. 2007;138:692.

2. Koh K-J, Kim K-A. Utility of the computed tomography indices on cone beam computed tomography images in the diagnosis of osteoporosis in women. Imaging Sci Dent. 2011;41:101-6.

3. Amam A, Rustom J. Assessment of mandibular alveolar bone density in osteoporotic adults in Syria. OJDOM. 2014;2:26-32.

4. Mostafa RA, Arnout EA, Abo el-Fotouh MM. Feasibility of cone beam computed tomography radiomorphometric analysis and fractal dimension in assessment of postmenopausal osteoporosis in correlation with dual X-ray absorptiometry. Dentomaxillofac Radiol. 2016;45:20160212.

5. Kazakia GJ, Majumdar S. New imaging technologies in the diagnosis of osteoporosis. Rev Endocr Metab Disord. 2006;7:67-74.

6. Knapp KM, Blake GM, Spector TD, Fogelman I. Can the WHO definition of osteoporosis be applied to multi-site axial transmission quantitative ultrasound? Osteoporos Int. 2004;15:367-74.

7. Parlani S, Nair P, Agrawal S, Chitumalla R, Beohar G. Role of Panoramic Radiographs in the Detection of Osteoporosis. Oral Hyg Health. 2014;2:2332-0702.1000121.

8. Marcus R, Majumder S. Osteoporosis. $2^{\text {nd }}$ ed. Burlington: Academic Press; 2001. p 3. 
9. Mohammed AR, Abbas FK, Hassan NA. Diagnostic efficacy of mandibular cortical thickness on panoramic radiographs to identify postmenopausal women with low bone mineral densities (Iraqi Population). J Am Sci. 2013;9:308-12.

10. Yashoda Devi B, Rakesh N, Ravleen N. Diagnostic efficacy of panoramic mandibular index to identify postmenopausal women with low bone mineral densities. J Clin Exp Dent. 2011;3:456-61.

11. Koh K-J, Kim K-A. Utility of the computed tomography indices on cone beam computed tomography images in the diagnosis of osteoporosis in women. Imaging Sci Dent. 2011;41:101-6.

12. Klemetti E, Kolmakov S, Heiskanen P, Vainio P, Lassila V. Panoramic mandibular index and bone mineral densities in postmenopausal women. Oral Surg Oral Med Oral Pathol. 1993;75:774-9.

13. Taguchi A, Tsuda M, Ohtsuka M, Kodama I, Sanada M, Nakamoto T, et al. Use of dental panoramic radiographs in identifying younger postmenopausal women with osteoporosis. Osteoporos Int. 2006;17:387-94.

14. De Vos W, Casselman J, Swennen GRJ. Cone-beam computerized tomography (CBCT) imaging of the oral and maxillofacial region: A systematic review of the literature. Int J Oral Maxillofac Surg. 2009;38:609-25.

15. White SC, Rudolph DJ. Alterations of the trabecular pattern of the jaws in patients with osteoporosis. Oral Surg Oral Med Oral Pathol Oral Radiol Endod. 1999;88:628-35.

16. Biostatistics DW. A foundation for analysis in the health science. $6^{\text {th }}$ ed. NY: John Wiley and Sons; 1995.

17. Barngkgei I, Al Haffar I, Khattab R. Osteoporosis prediction from the mandible using cone-beam computed tomography. Imaging Sci Dent. 2014;44:263-71.

18. Ledgerton D, Horner K, Devlin H, Worthington $H$. Radiomorphometric indices of the mandible in a British female population. Dentomaxillofac Radiol. 1999;28:17381.

19. Benson BW, Prihoda TJ, Glass BJ. Variations in adult cortical bone mass as measured by a panoramic mandibular index. Oral Surg Oral Med Oral Pathol.1991;71:349-56.

20. Delaney MF. Strategies for the prevention and treatment of osteoporosis during early postmenopause. Am J Obstet Gynecol. 2006;194:S12-S23.

21. Gomes C, de Rezende Barbosa G, Bello R, Bóscolo F, de Almeida S. A comparison of the mandibular index on panoramic and cross-sectional images from CBCT exams from osteoporosis risk group. Osteoporosis Int. 2014;25:1885-90.

22. Barngkgei I, Al Haffar I, Khattab R. Osteoporosis prediction from the mandible using cone-beam computed tomography. Imaging Sci Dent. 2014;44:263-71.

23. Melescanu-Imre M, Preoteasa E. Mandibular panoramic indexes predictors of skeletal osteoporosis for implant therapy. Curr Health Sci J. 2009;35:220-5.

24. Leite AF, de Souza Figueiredo PT, Guia CM, Melo NS, de Paula AP. Correlations between seven panoramic radiomorphometric indices and bone mineral density in postmenopausal women. Oral Surg Oral Med Oral Pathol Oral Radiol Endod. 2010;109:449-56.

25. Vlasiadis KZ, Skouteris CA, Velegrakis GA, Fragouli I, Neratzoulakis JM, Damilakis J, et al. Mandibular radiomorphometric measurements as indicators of possible osteoporosis in postmenopausal women. Maturitas. 2007;58:226-35.

26. Kim OS, Shin MH, Song IH, Lim IG, Yoon SJ, Kim OJ, et al. Digital panoramic radiographs are useful for diagnosis of osteoporosis in Korean postmenopausal women. Gerodontology. 2016;33:185-92.

27. Brasileiro C, Chalub L, Abreu M, Barreiros I, Amaral T, Kakehasi A, et al. Use of cone beam computed tomography in identifying postmenopausal women with osteoporosis. Arch Osteoporos. 2017;12:26.

28. Güngör E, Yildirim D, Çevik R. Evaluation of osteoporosis in jaw bones using cone beam CT and dual-energy X-ray absorptiometry. J Oral Sci. 2016;58:185-94.

29. Genant HK, Engelke K, Fuerst T, Glüer CC, Grampp S, Harris ST, et al. Noninvasive assessment of bone mineral and structure: state of the art. J Bone Miner Res. 1996;11:707-30.

30. Siris ES, Miller PD, Barrett-Connor E, Faulkner KG, Wehren LE, Abbott TA, et al. Identification and fracture outcomes of undiagnosed low bone mineral density in postmenopausal women: results from the National Osteoporosis Risk Assessment. JAMA. 2001;286:2815-22.

31. Hamdy RC, Petak SM, Lenchik L. Which central dual Xray absorptiometry skeletal sites and regions of interest should be used to determine the diagnosis of osteoporosis? J Clin Densitom. 2002;5:s11-s7.

32. Woodson G. Dual X-ray absorptiometry T-score concordance and discordance between hip and spine measurement sites. J Clin Densitom. 2000;3:319-24.

33. Singh SV, Aggarwal H, Gupta V, Kumar P, Tripathi A. Measurements in mandibular pantomographic X-rays and relation to skeletal mineral densitometric values. J Clin Densitom. 2016;19:255-61.

34. Passos JS, Gomes Filho IS, Sarmento VA, Sampaio DS, Gonçalves FP, Coelho JMF, et al. Women with low bone mineral density and dental panoramic radiography. Menopause. 2012;19:704-9.

35. Damilakis J, Vlasiadis K. Have panoramic indices the power to identify women with low BMD at the axial skeleton? Phys Med. 2011;27:39-43. 